\title{
Co-constructing Appreciative Inquiry across disciplines: A duo-ethnography
}

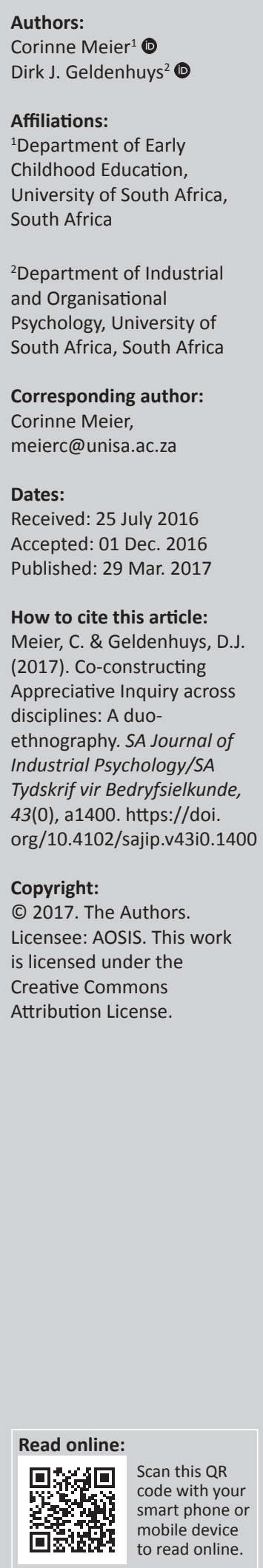

Orientation: Appreciative Inquiry (AI) has become increasingly popular as a tool for change management in the world of business and is spilling over into a range of contexts, linking a diversity of disciplines. However, instances where management has used AI in consultation with education for collaborative purposes could not be traced as yet.

Research purpose: The aim of this study was for two AI practitioners, one in the field of Industrial and Organisational Psychology and one in Education, to partake in a collaborative study proceeding from reflection on the said researchers' experiences with facilitating AI in different contexts.

Motivation for the study: With social constructionism as a core principle underlying AI, it is argued that sharing experiences across disciplines could enrich the literature and the application of $\mathrm{AI}$ in different contexts.

Research design, approach and method: The research is based on a qualitative, empirical, duo-ethnography using self-reflective narratives of the experiences of facilitating AI in crossdisciplinary contexts.

Main findings: Reflecting on experiences in various disciplines lead to the co-construction of new knowledge. Not only were similar experiences supported, validated and extended, thus affirming the strength-based principle of AI, but it also provided the opportunity for disciplinary cross-fertilisation by combining different perspectives regarding the formality of the AI process and the extent of the facilitator's and participants readiness to work with AI methodology.

Practical/managerial implications: The formality of the AI process and hence the extent of the facilitator's involvement (signalling his or her readiness to participate actively and take the lead in co-creating a new reality) must be tempered by due allowance for the participant's readiness to work with AI methodology. Furthermore, participants should be accommodated within the psychological space where they find themselves at the moment when the intended intervention is initiated.

Contribution/value-add: Duo-ethnography provided the researchers with the opportunity to challenge the 'other' to reflect on their own discipline-related AI experiences, in a deeper, more relational and authentic way. The voices and ideas identified and presented counter narratives, also blended in unique ways to augment the definition of AI as a multidisciplinary force to co-create a better society. More specifically, the 'readiness' of the facilitator for an AI encounter was conceptualised and applied to the psychological and behavioural readiness of not only the participants, but also the facilitators of AI workshops.

\section{Introduction}

With its focus on building on the positive and inclusive collaboration as founding concepts, Appreciative Inquiry (AI) has not only become increasingly popular as a tool for change management in the world of business for which it was originally intended, but is currently also applied in a variety of contexts and disciplines (Dematteo \& Reeves, 2010; Trajkovski, Schmied, Vickers \& Jackson, 2013). Application of this novel approach has yet to proceed beyond its infancy, particularly in the context of education (Cockell \& McArthus-Blair, 2012); therefore, the researchers argue in this article that sharing experiences of facilitating AI across contexts and disciplines may lead to the construction of enriched theoretical perspectives regarding the application of AI.

In this regard, it should be mentioned at the outset that the AI premise of building on the positive is counterintuitive, given the inveterate inclination of human nature to give precedence to the 
negative (Williams, 2014). Bauminster, Finkenauer and Vohs (2001) confirm that negative experiences or apprehensions take precedence over positive experiences in people's consciousness (i.e. in their basic mental predisposition). Rozin and Royzman (2001) state in corroboration that a negative disposition is more transmissible than a positive disposition. Similarly, Taylor (1991) explains that processing negative information requires a greater investment of neurological energy than that required to process positive information. The amygdala uses approximately two-thirds of its neurons to detect negative experiences, and any bad news is rapidly stored in the long-term memory, unlike positive experiences that have to occupy the recipient's consciousness for more than 12 seconds to secure transfer from short-term to longterm memory.

A recent study by Moser, Hartwig, Morgan, Jendrusina and Kross (2014) established the existence of brain markers that distinguish negative thinkers from positive thinkers. The research suggests that people can be broadly classified as positive and negative types. Seligman and Csikszentimihalyi (2014) contend that unless humans are occupied with specific thoughts, worrying is the brain's default position and that while negative emotion always has the ability to 'trump' positive emotion, individuals have to learn how to keep negative emotion in check by amplifying positive emotions. Moreover, Leaf (2013) takes the view that 'you have the power in your mind to change the negative ...'. Thus, considering that humans are inclined towards a negativity bias, the research question that arises is whether a negative bias influences the readiness of the facilitator and the participant in an AI encounter?

\section{Research purpose}

The inherent negative bias referred to above prompted two AI practitioners, one in the field of Industrial and Organisational Psychology (IOP) and one in education, to pioneer a collaborative study proceeding from reflection on the readiness of the said researchers' and the participants' readiness for encounters with AI methodology, given their negative inclination.

The next section provides the background on AI methodology as a theoretical framework for this research.

\section{Literature review}

$\mathrm{AI}$ is a collaborative, strength-based approach to transform human systems (e.g. companies, schools, organisations and institutions) towards a shared image of their most positive potential by first discovering the very best in their shared experience (Barrett \& Fry, 2005). AI is also defined as 'the cooperative co-evolutionary search for the best in people, their organisations, and the world around them' (Cooperrider, Whitney \& Stavros, 2008, p. 3). It is regarded not only as a way of being in the world but also as a methodology that is based on sound theoretical underpinnings, such as positive psychology with emphasis on the identification and maximisation of innate strengths as the key to optimising well-being, human flourishing and happiness (StrattonBerkessel, 2010).

The following five principles that give credence to links between theoretical developments across a range of disciplines such as organisational and learning theories, human development and interaction also serve as premises for AI (Barrett \& Fry, 2005; Cooperrider \& Whitney, 2005; Reed, 2007):

- The constructionist principle infers that words create worlds. Social constructionism with its defining characteristic that the world consists of relationships which we co-create is regarded as one of the core principles. What we focus on becomes our reality. Through conversations, people co-create the world or organisation they want to live in.

- According to the simultaneity principle, change starts with the very first question we ask in an AI encounter. The question we ask will determine the direction of the inquiry, emphasising the importance of unconditional, positive life-giving questions.

- The positive principle postulates that human systems are heliotropic ('helios' = sun), meaning that they tend to grow in the direction of (what is seen as) the source of life (Barrett \& Fry, 2005). It builds on constructs of humanistic psychology such as positive regard and supportive affect and their role in building or sustaining cooperative capacity. It is argued that positive questions in any encounter lead to deeper and more sustainable engagement.

- According to the anticipatory principle, the way people envisage the future will shape the way they move towards the future. As we anticipate the future, we create images of that future and then act as if that future were already happening. With reference to the placebo effect, Barret and Fry (2005, p. 48) state: 'Positive images of the future may be so powerful that they guide us at the cellular level'.

- The poetic principle states that what people focus on grow. Reality is created in the moment and there are multiple realities as people author their world, choosing those parts of the stories they want to focus on. People and organisations are like pieces of art with open-ended possibilities for interpretation, learning and inspiration. What we choose to inquire about opens up new possibilities for action. Thus, in choosing to focus on what works (the positive), we create a sense of possibility and hope.

With the above five principles which serve as premises for AI, a typical AI process (e.g. during a workshop) starts with the selection of a topic or a specific focus for the inquiry. Topics should be stated in the positive and be desirable, the participants should be curious and have a desire to learn more about the topic, and the topic should move in the direction the participants want to move (Reed, 2007). The process of selecting affirmative topics might include challenging people to reframe deficit issues into affirmative topics for inquiry 
(Cooperrider \& Whitney, 2005). The affirmative topics are formulated in questions, called an interview protocol or guide (Barrett \& Fry, 2005) and serves as the basis for the interviews of the discovery phase. The interview protocol typically includes general best experience questions to set the stage, followed by additional questions based on the identified topics, and ending with more open, externally focussed concluding questions such as wishes for the future. Negative experiences are often phrased as wishes in this part of the interview protocol (Reed, 2007; Watkins \& Mohr, 2001).

After the affirmative topic choice has been selected, the AI process follows the following phases, known as the 4-D cycle (Cooperrider \& Whitney, 2005):

- Discovery. The first phase of the cycle entails the mobilisation of all stakeholders in the identification, valuing and articulation of the 'best of what is' or the lifegiving forces or strengths of the system. This is done through interviewing in pairs, using predetermined interview protocols based on the affirmative topics. The next phases are normally done in group format.

- Dream. After the best of 'what is' has been discovered, conversations move towards 'what might be', entailing the envisioning of a preferred future and describing that future in a powerful purpose, vision or strategic intent or 'what is the world calling for' (Cooperrider \& Whitney, 2005, p. 16). During the dream phase, a convergence zone is created where the past meets the future.

- Design. This phase entails the co-creation of the social architecture of the system. Enabling mechanisms that will make the dream come true are identified by dialoguing what the ideal should be. Possibility statements are created through which the design of a future system is articulated. Like an architecture project, structures are created that become the containers and boundaries that facilitate action (Barrett \& Fry, 2005).

- Destiny. A process through which the new plans can emerge is developed during the destiny phase, thus enabling a whole system of sustainable development that can empower, impart knowledge and transform (Cooperrider \& Whitney, 2005, p. 16).

In summary, $\mathrm{AI}$ is a methodology that attends to the strengths or positive core of human systems (companies, schools, organisations and institutions). It follows, therefore, that the question to consider here is: If people are negatively inclined on balance, how would such a bias influence the readiness of the $\mathrm{AI}$ facilitator and the participant to participate in an AI intervention? This question is relevant as no research could be found where the application of AI was studied across disciplines and more specifically if people are negatively inclined on balance, how such a bias influences the readiness of the AI facilitator and the participant.

\section{Research design Research approach}

The research approach is grounded in a qualitative, empirical, duo-ethnography. The approach lies within how the qualitative methodology, specifically the self-reflective methodologies such as duo-ethnography and narrative enquiry, provides researchers the opportunity to delve into their own experiences (Silverman, 2013, p. 109) as AI practitioners in a quest to answer the research question. In this study, researchers practicing $\mathrm{AI}$ in different contexts (IOP and Education) juxtapose their AI histories to provide multiple understandings of their AI involvements. In this process, researchers reflect and reconceptualise perceptions of both themselves and their AI contexts (Norris, Sawyer \& Lund, 2012, p. 9).

\section{Research strategy}

Duo-ethnography is selected as an appropriate strategy for AI research. Both AI and duo-ethnography are concerned with uncovering the means people use to make sense of their realities (Reed, 2007). Having selected duo-ethnography as a strategy, the researchers embarked on a collaborative research journey in which they engaged in a dialogue on their distinct histories on a given phenomenon namely AI. The researchers' goal is to reflect, interrogate and reconceptualise existing beliefs about AI through a conversation that starts as a dialogue and concludes in written narrative format. Duoethnography as strategy was employed to demonstrate that through the articulation of contextual differences (IOP and Education), different people can experience the same phenomenon (AI) differently. Duo-ethnographers challenge the other to reflect on their own life in a deeper, more relational and authentic manner (Norris et al., 2012, pp. 10, 17).

\section{Research method}

The research method that is mostly associated with a duo-ethnography is self-reflective narratives and in this case self-reflective narratives of the experiences of facilitating AI in cross-disciplinary contexts. Walford (2004) labels self-narrative, personal narrative and auto-observation activities as essentially auto-ethnographic in nature, but according to Ellis and Bochner (2000) duo-ethnography draws on auto-ethnography and therefore uses self-reflective narratives in a collaborative way where two or more researchers engage in a dialogue on their distinct histories on a given phenomenon. In this dialogue, the voice and opinion of each duo-ethnographer is explicit. The individual self-reflective narratives end up in a scripted collage of interconnected ideas, which expands the possibilities for new insights (Norris et al., 2012, pp. 14,43) regarding the question how a negative bias would influence the readiness of the AI facilitator and the participant.

\section{Research setting}

Four case studies (interventions or workshops) were reflected on, two from business settings and two from educational settings. The first two interventions were facilitated by an industrial psychologist, and the other two interventions were facilitated by an educational specialist. The one business setting was an international company in the tourist industry, and the other setting was a small business within the engineering industry. Regarding the educational setting, 
one intervention was facilitated in a department of a tertiary institution, and the other intervention was facilitated in a primary school.

\section{Research participants and sampling methods}

In a duo-ethnography, the participants in the research refer to the researchers. In this study, the hybrid identities of the researchers originate from the designated disciplinary contexts from which the researchers engaged namely IOP and Education. The researchers use their own biographies from their respective disciplines as sites of research.

Purposive sampling was used. The sampling of the case studies was done in such a manner as to reflect different contexts as well as facilitators from different disciplines.

\section{Data collection and recording}

The collection of data and the recording thereof in duo-ethnographic studies are not prescriptive (Norris et al., 2012, p. 139). Furthermore, the steps of data collection in qualitative research are fluid in the sense that data collection is not always sequential to or separate from that of data analysis and interpretation. The data collection process is often interweaved and interactive with data analysis and interpretation (Chang, 2007). Following the mentioned interactive character of data collection and recording, the two researchers attempted to balance expression of views and the explanation thereof (Norris et al., 2012, p. 32). The expression of views was done through a dialogue between the researchers on their distinct histories on facilitating AI sessions. The explanation was compiled by interrogation of the self and the other in an effort to re-conceptualise existing beliefs on AI. Thus, the reflection were first done individually, then together and thereafter the different experiences were juxtaposed.

The dialogue or conversations in the expression and explanation phases were written in the form of self-reflective narratives. The self-reflective narratives are characterised by a back-and-forth movement (Norris et al., 2012, p. 44) as the researchers tried to make meaning of their own and the others perspective on the same phenomenon which is AI.

\section{Strategies employed to ensure data quality and integrity}

According to Norris et al. (2012, p. 20), the 'believability and trustworthiness of duo-ethnographical research is found in the depth of researcher involvement'. The researchers intentionally created transparency and articulation of perspectives, thoughts and wonderings, by purposefully creating self-reflexive reconstruction (Sameshima, 2013) of their own experiences in facilitating AI workshops.

\section{Data analysis}

The self-reflexive reconstruction of perspectives and thoughts of the researchers followed the following sequence: firstly, individual analytic and self-reflective narratives were generated on AI encounters in IOP and educational contexts and secondly dialogic face-to-face communication, over a period of time, across the two disciplines followed, which produced narratives in which new knowledge or awareness of the researchers and their participants' readiness to engage in AI methodology were captured. The narratives were thematically analysed and the themes were labelled as 'lessons learned' from engaging in AI through a duoethnographic lens.

\section{Reporting style}

The confluence of narratives will be precluded by individual narratives from the IOP context followed by the educational context. In order to distinguish between the narratives of the industrial psychologist and the educational specialist, the individual narratives will be introduced accordingly. All the results will be reported in the first person.

What is to follow is a summary of the researchers' dialogical process of making meaning of the readiness for the involvement of the facilitator and the participant in AI methodology.

\section{Findings}

The themes will be offered in the form of individual lessons that were learnt. Through collaboratively juxtaposing the lessons, further reflection will be offered thereafter.

\section{Lesson 1: Appreciative Inquiry as a change methodology of choice \\ Industrial psychologist}

I chose to do my doctorate studies on the contribution of systems-psychodynamic interventions to organisation development. My motivation was that my training for the master's degree did not provide me with enough 'depth' to make sense of the irrational behaviour of organisations, especially during times of turmoil and transformation. Systems psychodynamics, with its focus on the unconscious irrational dynamics of human systems, and AI with its focus on the positive can almost be regarded as two opposing interventions, based on two opposing paradigms in psychology, namely psychodynamics and positive psychology.

Before completing my doctoral studies, I got introduced to AI at a workshop presented by Gervase Bushe during a visit to our university. As I was already forming my research identity around psychodynamics, I did not find this 'superficial' approach called AI convincing. However, on completion of my doctorate, I attended a number of AI workshops presented by Professor Freddie Crous, a colleague and friend of mine from another tertiary institution. In hindsight, I am of the opinion that it was not the AI methodology as such that attracted me in the first instance, but the personal manner in which he lived AI as facilitator, colleague and friend, that convinced me of the possibility of using $\mathrm{AI}$ as an alternative to what I endorsed over a couple of years. Although I practiced both, I was also looking for a way to integrate the two 
opposing paradigms. The more exposure to and reflection on positive psychology eventually got the better of me regarding my use of methodology for change interventions. But it was only until I ventured reading on neurosciences that I could finally endorse AI as my methodology of choice, also preferring training students in $\mathrm{AI}$ and facilitating $\mathrm{AI}$ workshops for clients.

\section{Lesson 2: Appreciative Inquiry as fulfilling human needs of love, care and belonging}

\section{Education specialist}

All I ever wanted to do in life since I was little was to be a teacher. I qualified as a teacher in Early Childhood Education (ECE) some decades ago and steered my ECE journey to its current port as lecturer in ECE at a tertiary institution. After many engagements with literature and practice in the field of ECE, it dawned on me when preparing my inaugural lecture with the theme 'Windows of opportunity, use it or lose it: Early Childhood Development prospects in South Africa' (Meier, 2014) that the notion of 'care' lies as the underpinning in my heart and soul when I reflect on children and the education of children. The 'ethics of care' concept was coined by Noddings (1984) who argues that caring should be the foundation for decision-making in the lives of children.

The young child in the ECE phase is in the most susceptible stage of developmental stages especially brain development. Longstanding as well as recent theories and research done on child development are most informative as regards windows of opportunity for the young child. The young child is, however, dependent on someone who cares enough to open these windows of opportunity so that the child can step out and explore the world on a road to live up to realising their full potential.

The road to realising one's full potential is structured according to Maslow's hierarchy of five interdependent levels of basic human needs (motivators) that must be satisfied in a sequence starting with the lowest level. Physiological needs for survival (to stay alive and reproduce) and security (to feel safe) are the most fundamental and most pressing needs. They are followed by social needs (for love, care and belonging) and a need for self-esteem (to feel worthy, respected and have status). The final and highest needs are those for self-actualisation (self-fulfilment and achievement) (Maslow, 1954).

It was after a conversation with my co-researcher in the IOP field about AI that I realised that the two drivers in my personal and academic life namely 'care' and Maslow's third level of human needs namely 'love, care and belonging' link very well with AI. I just had to know more about AI and subsequently attended a world conference on $\mathrm{AI}$ as well as registered for a certificate course in AI. As part of the practical component of the certificate course, I had to design and conduct a workshop based on AI methodology. My colleagues in a teacher training department formed the context for the workshop. In my planning for the workshop, I infused the concept of care and Maslow's theory with AI methodology. One of the principles of $\mathrm{Al}$ namely social constructionism with its defining characteristic that the world consists of relationships which we co-create resonates very well with the concept 'care'.

Inspired by the training in AI, I conducted a second workshop based on AI methodology. This time I shared AI methodology, Maslow and 'care' with a group of 12-year-old prefects at a primary school. AI methodology suited the engagement with the prefects perfectly, as the AI principles namely the simultaneity, poetic, anticipatory and positive principles (as discussed in the literature review on AI methodology) build on constructs such as positive regard and supportive affect (care) and their role in building or sustaining co-operative capacity. All the above AI principles will serve the prefects well as they are at the beginning of life's journey, and a positive attitude will shape the way they move towards the future.

\section{Lesson 3: A negative bias assists facilitation} Industrial psychologist

After reflecting on the above, my initial discovery was that I was probably not ready for a positive, strength-based approach to human behaviour and organisational interventions when encountering AI, because of my background in systemspsychodynamics. I was not really embracing AI as a living philosophy. However, my discovery phase continued as is evident from my reflection on the following example of an AI workshop a colleague and I facilitated in the tourism industry.

A colleague and myself facilitated an AI as part of a programme to train the organisational effectiveness team of a large international company who envisaged using $\mathrm{AI}$ as methodology to merge different organisational cultures. A participant, trained as a psychologist, who contracted with us for the training, previously attended an AI workshop but did not have formal training in facilitating an AI. She was also a participant in the workshop. As with my previous experiences, most of the participants were mostly neutral at the beginning of the workshop - accept for the psychologist who were very positive from the start, they became more positive as the process unfolded. One participant, however, played the devil's advocate throughout the workshop, challenging the approach as not really suitable for her team. She, holding a master's degree in Business Administration, was responsible for establishing a training culture in the company. She informed us that she is working with difficult managers who seldom comply with the HR strategies she has developed for the company and that a more disciplined approach is necessary. We also realised that she was appointed in her role because of her strength in implementing strategies in similar difficult environments. Attempts were made during the workshop by co-participants to convince her of the feasibility of using AI, even in her context. This group dynamic was, according to my interpretation, to some extent led by the psychologist. 
My analysis of her resistance was that this particular participant was not ready for AI, possibly because she identified strongly with the constructed reality of being competent in her approach that led to her being offered her the current role she occupied, and, or because she could have felt being manipulated during the session. She would have found it very difficult to embrace positivity as living philosophy.

As I reflected on my own role as facilitator, I caught myself focussing on the so-called 'negative participant' and not on the profound learning that took place for all the other participants. Furthermore, I was also interpreting behaviour, instead of displaying curiosity with an appreciative eye. As a result of this realisation, I started questioning my own readiness as facilitator for an AI session. Through further reflection, however, I came to realise that as $\mathrm{AI}$ is based on social constructionism, opposing viewpoints should also be appreciated, even if these viewpoints could be seen us unappreciative. This reflection led to an insight that taught me a valuable lesson. Without my problem-focussed predisposition, I would probably not have been able to empathise with the participant's experience. What I thought of as a disqualifier became a facilitator's strength by default. This insight helped me to interpret a number of other AI interventions to good effect where there was a wish for buyin for all the participants.

The following example serves to illustrate my sense making of AI as methodology based on us sharing our experiences as facilitators. I was invited to facilitate an AI intervention for the management team of a small company for strategic planning purposes. The team consisted of six members, including the founder (aged 72) and his stepson (aged 28 old). Although all team members agreed that the approach was positive it turned out that the father and son were at loggerheads and ironically had to interview each other during the discovery phase of the intervention. Furthermore, the founder strongly disapproved of the printed interview protocol, stating that he resented all paperwork. I therefore had to intervene in this awkward situation and facilitate the discussion between the two members.

The questions raised by this situation were:

Was this pair ready for the intervention? Can a person who started a successful company be so change-resistant, or could communication between father and son be so dysfunctional that AI was inappropriate? Was the participant system ready for the intervention?

However, one sentence uttered by the stepson saved the workshop (or possibly even the future of the company): 'My wish is that you will never retire because I can learn so much from you!' In light of my awareness of the transformative power of AI, I was intrigued by the founder's resistance to using the AI protocol for storytelling during the discovery phase. On reflecting on the experience, I realised that resistance is not only against the negative, it is not about fearing the potential loss of something positive, it is about the unknown, the newness of a different experience, it is about change per se, even if the change is (foreseeably?) for the better.

\section{Lesson 4: Not always using an Appreciative Inquiry protocol assists facilitation}

\section{Education specialist}

During the planning of the workshops for the colleagues in a teacher training department and the prefects at the primary school I used AI methodology, including Maslow's hierarchy of human needs and 'care' as guiding principles. I was rather nervous to facilitate the workshops as I was actually a newcomer to AI methodology, but my experience as a lecturer in the field of education and my personal positive attitude to life gave me the confidence to push through. Although I have taken the context (age, gender, life experiences) of each group into consideration when I prepared the workshop material, I made some vital discoveries during the course of the workshops. For instance, during the workshop with the colleagues in the teacher training department, I felt as if the workshop never found the momentum I anticipated. Reflecting on this I realised that the group, being teacher trainers, probably routinely applied the AI and 'care' principles in performing their daily training task, with the result that the workshop may not have added anything new to their lives. Although the prefects were excited about the workshop as an extramural activity and a lot of energy was generated by the workshop activities and materials I, as workshop facilitator, had to change these activities and the interview protocol more than I thought would be necessary during the course of the workshop in an effort to sustain high-level input. Both workshops ended on a high note however, and feedback from the participants reflected positive life-enhancing outcomes.

In reflection on my feelings on the outcome of both workshops, I discovered that as facilitator of AI-based workshops I was not quite ready to facilitate AI methodology. In the case of the teacher educator workshop, I tried to keep strictly to the methodology of AI and tried somewhat overenthusiastically to infuse a positive attitude to life into already positive participants. In essence, I was not ready to accept the possibility that not all participants in an AI workshop are negatively inclined by nature. In the case of the prefect workshop, I again tried to keep strictly to the AI methodology but realised that the participants were not ready, on a developmental level, to embrace the structured way of facilitating an AI workshop.

On further reflection, I realised that for lack of apposite experience I was also not ready to use the AI methodology, with special reference to the AI protocol. Consequently, I realised that what I had considered a shortcoming turned out to be an advantage as the children were also not ready to use the formal AI methodology - especially the AI protocol.

Whereas the previous sections mainly focussed on the self-reflections of each researcher, these following sections 
represent a co-construction and interpretation of the selfreflections.

\section{Further reflection: Juxtaposing content and experiences}

Juxtaposing our narratives led us to the discovery of the following. Although we both realised that we have a common understanding of and embracing $\mathrm{AI}$ as a collaborative, positive, strength-based approach to develop and transform human systems (Barrett \& Fry, 2005), our background as facilitators influenced our experiences and hence our preferences for facilitation of an AI process. We became aware of how our different disciplines influenced our focus on AI as facilitators. The role of the educational specialist is influenced by her inclination to focus on the development of children, while the industrial psychologist's focus is on AI methodology as a positive approach to change management. From an educational perspective, the emphasis was on living AI (a way of being in the world), while the industrial psychologist was more concerned about using AI methodology for transforming human systems, more specifically in the work context. Furthermore, the industrial psychologist's background in systems psychodynamics influenced him to analyse the behaviour of participants in a workshop, while the educational specialist was influenced by her bias towards the positive, to focus on developing a positive bias in participants' outlook on life.

However, we both experienced that participants were occasionally reluctant or resistant to AI methodology with its unconditional focus on the positive, especially at the outset, not only because of an inherent negative bias but in reaction against the formality of the methodology, with particular reference to the interview protocol. This happened with a participant with more than 70 years of life experience as well as a teenager at the age of 12 , regardless of their possible bias towards the negative or positive. Their experiences seem to be similar. For example, their demeanour was to some extent resistant even at the prospect of positive change.

Through reflection on our narratives, we both realised that what we considered to be our shortcoming as facilitators, because of our backgrounds, could be reframed as our strengths. For instance, it helped us to appreciate participants who were not positive towards AI and therefore enabled us to meet them in the psychological space where they were situated at the time as regards their world view and professional background. We could also more readily accommodate participants who were prepared to engage with formal AI methodology in that we could assess their readiness to assume their life's journey in an appreciative frame of mind, besides which we could fine-tune the AI process while it was in progress in order to benefit their specific disposition.

We found that both of us embraced AI as a process of continuous learning, commencing at different places and times in our professional careers as well as our lives, but that we also mutually influenced one another in terms of developing skills in the refinement of AI methodology, as well as in embarking on an appreciative life journey. Although we realised that the learning happened through individual self-reflection and mutual reflection (evidenced by our narratives), we could not clearly distinguish between learning occasioned by individual or mutually interactive reflection.

We also found that as facilitators from different backgrounds and disciplines, our respective learning experiences could effectively become a joint transformative force that could work together towards creating a better society. Instead of creating disparate views on what $\mathrm{AI}$ is and how it should be facilitated, the different backgrounds and disciplines may lead to a confluence of narratives through which we came to realise that there is no right or wrong and that the terms in which AI is defined in the literature make provision for AI as a living philosophy as well as implementation of its methodology.

It should be noted further that the said confluence of perspectives enables facilitation of AI to accommodate a divergent population of participants ranging from children to the elderly, from participants from society at large to participants in the workplace, from participants embracing the positive in life to participants who are used to injurious experiences, especially in the workplace, and who have therefore been negatively attuned to the vicissitudes of life by conditioning. This happens formally (using clear AI methodology), as well as informally, depending on participants' readiness.

\section{Discussion}

The aim of this study was to report on an empirical duoethnography using self-reflective narratives of our experiences of facilitating AI workshops across disciplines and contexts.

To distil the meaning of our findings, we decided to use the construct of readiness for change as a yardstick. Readiness for change is a multilevel and multifaceted construct that refers to a state of being both psychologically and behaviourally ready (display the necessary ability) to take action (Sekerka, Zolin \& Smith, 2009). The construct is conceptualised in the literature from an individual as well as an organisational perspective with the focus on the employee or participants, especially during the first phase of a change management intervention (Sekerka, Brumbaugh, Rosa \& Cooperrider, 2006). The construct of change readiness is also applied to classify change interventions according to deficit versus strength-based approaches (Sekerka et al., 2009).

All participants in an AI workshop cannot be regarded as psychologically ready for the intervention in terms of the above-mentioned definition. Given people's naturally negative inclination (e.g. manifesting as fear of negative consequences) 
(Baumeister, Finkenauer \& Vohs, 2001; Williams, 2014), AI-based interventions with their exclusive focus on positive, strength-based experiences can be met with resistance by participants who are negatively inclined. These participants may experience the process, especially in the beginning, as unsafe as they are not allowed to speak about their negative experiences. Neuroscientific research has indicated that allowing people to label their negative feelings enables them to manage their emotional experiences and hence to contribute to the experience of safety (Henson \& Rossouw, 2013). The experience of being unsafe may be induced by the shockingly counterintuitive novelty of exposure to the AI methodology. Human beings are often not aware of how their expectations, which are often negative, influence their perceptions and even their behaviour in the present moment (Henson \& Rossouw, 2013).

Participants' readiness is also influenced by their ability to engage in a formal AI process, despite their age and discipline. Even people with a positive bias towards life may be hesitant to engage with AI, especially in the beginning phase of the process, because of their lack of readiness for the exposure to the formal AI process. This finding supports educational research identifying note taking, probing, technical difficulties, nervousness, time restrictions and interviewing friends as some of the difficulties experienced by children during an AI process (Shuayb, Sharp, Judkins \& Hetherington, 2009). Readiness for AI, therefore, implies a choice for the positive; that is a willingness to embrace appreciation (as a way of life) and skills (methodology) to transform realities.

Similar to the readiness of the participant, the readiness of the facilitator also plays a significant role in facilitating an AI process. Readiness to facilitate a positive, strength-based collaborative process thus also entails the facilitator's psychological readiness (his or her frame of reference) as well as his or her acquired practical ability to apply the methodology. This is in line with defining AI as a living philosophy and a methodology (Watkins \& Mohr, 2001).

The influence exerted by the facilitator's credibility and just appreciation of participants' state of readiness for change is acknowledged in the literature (Sekerka et al., 2009). Regarding AI, however, we argue that a deficit-based background is not necessarily a weakness as it might facilitate positive change for a participant who is negatively inclined and thereby persuade the participant that it is safe to participate in the AI process. This might happen, probably because the participant finds it possible to identify with the facilitator. This seems to be in line with the poetic principle of AI according to which people are like pieces of art with openended possibilities for interpretation, learning and inspiration (Cooperrider \& Whitney, 2005; Reed, 2007). Again, facilitators' readiness to apply AI methodology influences the success or otherwise of applying AI. Facilitators with a positive bias can successfully adapt the methodology to accommodate the readiness of the participants, even if the facilitator is not well trained and experienced in the formal AI process.
Instead of a prerequisite for inducing positive change via AI, readiness for change is considered a process (i.e. transition from not ready to ready). This is evident from the learning experiences during individual self-reflection and joint giveand-take reflection by researchers according to their respective narratives. Readiness to facilitate AI sessions, therefore, depends on facilitators' exposure, for example to number of sessions facilitated, number of workshops attended and degree of reflection on AI in the course of workshops. AI can thus be regarded as a methodology for facilitating readiness to apply appreciation as a living principle of a living philosophy.

Furthermore, readiness can be viewed as a mutual process that is not only influenced by the co-construction of new realities by the participants but also between the participants and the facilitator and between co-facilitators. For instance, the facilitator's capacity to envisage the maximum potential and future possibilities of negatively inclined participants will help the facilitator and participants to become a joint transformative force. The social constructionism principle underlying $\mathrm{AI}$ is therefore extended to the relationship between the participant and the facilitator as well as the relationship between the facilitators.

Based on the above, our findings could be used to augment the definition of $\mathrm{AI}$ as a multidisciplinary force to co-create a better society. We, therefore, propose a view of AI as a choice for a positive, transformative way of being, implemented through accessible methodology by various facilitators in conjunction with participants, young and old, proceeding from an extended range of mind sets, who are ready to co-create a desired future for all. It stands to reason that the diversity of mind sets brought to the situation will have originated from a diversity of social realities that should be acknowledged and dialogued in order to enrich the theoretical perspectives on $\mathrm{AI}$ as a methodology and a way of being. Accommodation of diversity in this way amounts to an extension of the role of the facilitators in obedience to the principle of social constructionism whereby people co-create the world they want to live in through conversations.

\section{Practical implications}

The findings of the inquiry under review have implications not only for the facilitation of AI workshops across disciplines and contexts but also for AI practice. In order to facilitate change towards searching for the positive, participants should be accommodated within the psychological space where they find themselves at the moment when the intended intervention is initiated. For example, such accommodative intervention could include listening to and validating the negative narratives offered by participants before choosing a topic of affirmative purport. Reframing, that is normally used before the first phase of an AI intervention (Cooperrider \& Whitney, 2005) to determine the topic, should not only be seen as a preliminary that precedes intervention but could also be part of an actual process of intervention (or even a standalone $\mathrm{AI}$ ) with an affirmative topic as its outcome. Unconditional positive 
regard can be regarded as a first step in the direction of an appreciative way of life. It may even be necessary to facilitate the process on an individual basis by means of appreciative coaching (Clancy, Binkert \& Orem, 2007).

Furthermore, the formality of the process and hence the extent of the facilitator's involvement (signalling his or her readiness to participate actively and take the lead in cocreating a new reality) must be tempered by due allowance for the participant's readiness to work with AI methodology. Mechanistic (i.e. 'by the book') application of formal AI methodology without improvising for particular circumstances must be avoided for the sake of participants who find it difficult to apply the formal AI methodology, specifically the interview protocol.

\section{Limitations and recommendations}

Finally, the use of only four cases, two per researcher, as examples to base our hypotheses on can be seen as a limitation of the study. It is recommended that narratives not only of facilitators but also of participants of AI interventions be studied in order to augment and to counter our assumptions.

\section{Conclusion}

Duo-ethnography as strategy provided the researchers with the opportunity to challenge the 'other' to reflect on their own discipline-related AI experiences, in a deeper, more relational and authentic way. It became clear that when duoethnographers collaborate, their voices and ideas blend in unique ways. On the other hand, duo-ethnography also provides a method to identify and present counter narratives. It is evident from the narratives that experiences in various disciplines lead to the co-creation of new knowledge. Not only were similar experiences supported, validated and extended, thus affirming the strength-based principle of $\mathrm{AI}$, but it also provided the opportunity for disciplinary crossfertilisation by combining different perspectives regarding the formality of the AI process and the extent of the facilitator's and participants readiness to work with AI methodology.

\section{Acknowledgements}

We acknowledge all participants who attended our interventions for allowing us to reflect on and to report our narratives on our collaborative learning.

\section{Competing interests}

The authors declare that they have no financial or personal relationship(s) which may have inappropriately influenced them in writing this article.

\section{Authors' contributions}

C.M. and D.J.G were co-responsible for doing the research and writing the article.

\section{References}

Barrett, F.T., \& Fry, R.E. (2005). Appreciative Inquiry: A positive approach to building cooperative capacity. Chagrin Falls, OH: Taos.

Baumeister, R.F., Finkenauer, C., \& Vohs, K.D. (2001). Bad is stronger than good. Review of General Psychology, 5(4), 323-370. https://doi.org/10.1037/10892680.5.4.323

Chang, H. (2007). Autoethnography: Raising cultural consciousness of self and others. In G. Walford (Ed.), Methodological developments in ethnography: Studies in educational ethnography, vol. 12, pp. 207-221. Boston: Elsevier.

Clancy, A.L., Binkert, J., \& Orem, S. (2007). Appreciative coaching: A positive process for change. San Francisco, CA: Jossey Bass.

Cockell, J., \& McArthur-Blair, J. (2012). Appreciative Inquiry in higher education: A transformative force. San Francisco, CA: Jossey-Bass.

Cooperrider, D.L., \& Whitney, D. (2005). Appreciative Inquiry: A positive revolution in change. San Francisco, CA: Barrett-Koeheler.

Cooperrider, D.L., Whitney, D., \& Stavros, J.M. (2008). Appreciative Inquiry handbook (2nd edn.). Brunswick, OH: Crown Publishing.

Dematteo, D., \& Reeves, S. (2010). A critical examination of the role of Appreciative Inquiry within an interprofessional education initiative. Journal of Interprofessional Care, 25(3), 203-208.https://doi.org/10.3109/13561820.2010. 504312

Ellis, C., \& Bochner, A.P. (2000). Autoethnography, personal narrative, reflexivity: Researcher as subject. In N.K. Denzin \& Y.S. Lincon (Eds.), The SAGE book of qualitative research (2nd edn., pp. 733-768). Thousand Oaks, CA: Sage.

Henson, C., \& Rossouw, P. (2013). Brain wise leadership: Practical neuroscience to survive and thrive at work. Sydney: Learning Quest.

Leaf, C. (2013). Switch on your brain. The key to peak happiness, thinking and health Grand Rapids, MI: BakerBooks.

Maslow, A. (1954). Motivation and personality. New York: Harper.

Meier, C. (2014). Windows of opportunity: Early childhood development prospects in South Africa. Journal of Social Sciences, 40(2), 159-168.

Moser, J.S., Hartwig, R., Morgan, T.P., Jendrusina, A.A., \& Kross, E. (2014). Neura markers of positive reappraisal and their associations with trait reappraisal and worry. Journal of Abnormal Psychology, 123(1), 91-105. https://doi.org/10.1037/ a0035817

Noddings, N. (1984). Caring. A feminine approach to ethics and moral education. Berkeley, CA: University of California Press.

Norris, J., Sawyer, R.D., \& Lund, D.E. (Eds.). (2012). Duoethnography: Dialogic methods for social, health, and educational research. Walnut Creek, CA: Left Coast Press.

Reed, J. (2007). Appreciative Inquiry: Research for change. Thousand Oaks, CA: Sage.

Rozin, P., \& Royzman, E.B. (2001). Negativity bias, negativity dominance, and contagion. Personality and Social Psychology Review, 5(4), 296-320. https://doi. org/10.1207/S15327957PSPR0504_2

Sameshima, P. (2013). Duoethnography. Understanding qualitative research \& duoethnography: Promoting personal and societal change within dialogic self study. Journal of the Canadian Association for Curriculum studies, 11(1), 1-20.

Sekerka, L.E., Brumbaugh, A., Rosa, J., \& Cooperrider, D. (2006). Comparing Appreciative Inquiry to a diagnostic technique in organizational change: The moderating effects of gender. International Journal of Organization Theory and moderating effects of gend
Behavior, 9(4), 449-489.

Sekerka, L.E., Zolin, R., \& Smith, J.G. (2009). Be careful what you ask for: How inquiry strategy influences readiness mode. Organizational Management Journal, 6, 106-122. https://doi.org/10.1057/omj.2009.15

Seligman, M.E.P., \& Csikszentmihalyi, M. (2014). Flow and the foundations of positive psychology. In M.E.P. Seligman \& M. Csikszentmihalyi (Eds.), Positive psychology: An introduction (pp. 279-298). Dordrecht, Netherlands: Springer.

Shuayb, M., Sharp, C., Judkins, M., \& Hetherington, M. (2009). Using Appreciative Inquiry in educational research: Possibilities and limitations. Report. Slough: NFER.

Silverman, D. (2013). Doing qualitative research (4th edn.). London: Sage.

Stratton-Berkessel, R. (2010). Appreciative Inquiry for collaborative solutions. San Francisco, CA: Wiley.

Taylor, S.E. (1991). Asymmetrical effects of positive and negative events: The mobilization-minimization hypothesis. Psychological Bulletin, 110(1), 67-85. https://doi.org/10.1037/0033-2909.110.1.67

Trajkovski, S., Schmied, V., Vickers, M., \& Jackson, D. (2013). Implementing the 4D cycle of Appreciative Inquiry in health care: A methodological review. Journal of Advanced Nursing, 69(6), 1224-1234. https://doi.org/10.1111/jan.12086

Walford, G. (2004). Finding the limits: Autoethnography and being and Oxford University Proctor. Qualitative Research, 4(3), 403-417. https://doi. org/10.1177/1468794104047238

Watkins, J.M., \& Mohr, B.J. (2001). Appreciative Inquiry: Change at the speed of imagination. San Francisco, CA: Jossey-Bass.

Williams, R. (2014). Are we hardwired to be positive or negative? On the capacity to emphasize the negative rather than the positive. Retrieved May 10, 2016, from https://www.psychologytoday.com/blog/wired-success/201406/are-wehardwired-be-positive-or-negative 